

\title{
Rate adaptation scheme for IEEE 802.11-based MANETs
}

\author{
Abderrahim Benslimane, Abderrezak Rachedi
}

\section{To cite this version:}

Abderrahim Benslimane, Abderrezak Rachedi. Rate adaptation scheme for IEEE 802.11-based MANETs. Journal of Network and Computer Applications (JNCA), 2014, 39 (1), pp.126-139. 10.1016/j.jnca.2013.05.013 . hal-00828494

\section{HAL Id: hal-00828494 \\ https://hal.science/hal-00828494}

Submitted on 31 May 2013

HAL is a multi-disciplinary open access archive for the deposit and dissemination of scientific research documents, whether they are published or not. The documents may come from teaching and research institutions in France or abroad, or from public or private research centers.
L'archive ouverte pluridisciplinaire HAL, est destinée au dépôt et à la diffusion de documents scientifiques de niveau recherche, publiés ou non, émanant des établissements d'enseignement et de recherche français ou étrangers, des laboratoires publics ou privés. 


\title{
Rate Adaptation scheme for IEEE 802.11-based MANETs
}

\author{
Abderrahim Benslimane $^{\mathrm{a}}$, Abderrezak Rachedi ${ }^{\mathrm{b}}$ \\ ${ }^{a}$ University of Avignon, Computer Science Laboratory of Avignon (LIA) \\ 84911 Avignon cedex 9, France \\ E-mail: abderrahim.benslimane@univ-avignon.fr \\ ${ }^{b}$ University of Paris-Est, Gaspard Monge Computer Science Laboratory (LIGM-UMR 8049), \\ 77454 Marne-la-Valle, France \\ E-mail: rachedi@univ-mlv.fr
}

\begin{abstract}
Rate adaptation is a highly challenging task in MANETs, mainly when relative fairness among competitive nodes is considered. Existing rate adaptation solutions are mainly designed for IEEE802.11-based WLANs. They do not cope with relative fairness. Unlike these existing schemes, the main objectives of our proposed approach, called REFOT (Relative fairness and Optimized Throughput), are to ensure fairness and to allow each node to adapt its transmission rate and contention window to its channel quality. The channel quality is determined by calculating for each node the probability to access the channel in a distributed manner by approximating the number of successful and failed transmissions. REFOT allows for reaching the appropriate transmission rate level, without crossing all the intermediate levels. This operation helps in avoiding scenarios where the network capacity could be underutilized or overused, allowing the system to reach its stability faster. We validate the proposed model via analytical model, based on a 3-dimension Markov chain, and simulation results. Via extensive simulations, the performance of REFOT is evaluated and compared against that of some existing schemes. In the performance evaluation, different node densities, mobility models, transmission ranges and network TCP/UDP traffic loads are simulated. The obtained simulation results are encouraging and indicate that REFOT achieves its design goals: it ensures a good trade-off between fairness and throughput.
\end{abstract}

Keywords: MANET, IEEE 802.11, DCF, fairness, throughput and Markov Chain.

\section{Introduction}

The IEEE 802.11 technology implements different Medium Access Control (MAC) methods for both centralized (e.g., wireless LAN) and ad hoc networks. The Distributed Coordination Function (DCF) is the fundamental MAC technique of IEEE 802.11 [11]. It is based on the Carrier-Sense Multiple Access and Collision Avoidance (CSMA/CA) scheme. IEEE 802.11 provides various transmission rates. For instance, in IEEE 802.11a, discrete rates are available ranging from $6 M b p s$ to $54 M b p s$, whereas in IEEE $802.11 b$, four transmission rates are available (i.e., $\{1,2,5.5,11\} \mathrm{Mbps})$. Although we have various transmission rates available in IEEE 802.11, there is no standard approach defined to select the appropriate rate while ensuring fairness among the competing nodes. Indeed, fairness and throughput optimization have never been jointly addressed. With this regard, it should be noted that without ensuring an acceptable level of fairness, the whole network becomes unable to reach its optimum cooperative status.

In the recent literature, various rate adaptation schemes have been proposed for WLAN networks. Auto Rate Fallback (ARF) [28] and Collision-Aware Rate Adaptation (CARA) [17] are few notable examples. However, these schemes are not applicable, in their current format, to Mobile Ad hoc Networks (MANETs). Effectively, unlike WLAN which acquires a centralized control unit, MANET networks lack such unit; which renders the fairness issue an important challenge in case of MANETs. As a matter of fact, a MANET node cannot adapt its rate without taking into account the other competitive nodes. Moreover, competing nodes do not necessarily have the same channel conditions. They may, therefore, experience different channel qualities. If a given node does not take into account its competitive neighbors in its rate adaptation operation, an unfair situation is likely to occur.

IEEE 802.11 standard does not take into account fairness in the context of MANET. Although the standard presents various transmission rates, it does not specify how to efficiently allocate these rates. Generally speaking, the effectiveness of a rate adaptation scheme hinges on how it is coping with the impact of transmission failures which may occur due to channel errors or packet collisions. In the literature, a wide set of rate adaptation schemes have been proposed $[8,28,10,15]$. Unfortunately, none of them is applicable to MANETs. The Receiver-Based Auto Rate (RBAR) scheme [10] is based on SNR values whereby a receiver chooses the next rate for its corresponding sender. However, the receiver may not have a correct interpreta- 
tion of the sender channel and other competitive nodes of the sender. Hence, it does not take into account fairness among competing nodes. In another scheme, called Automatic Rate Fall-back (ARF) [28], a sender deduces the channel condition by measuring consecutive successful and failed transmissions. The sender then adjusts its rate in accordance with them. However, the drawback of this scheme is that the sender does not care about other competing nodes and adapts its rate without taking them into account. Despite its wide usage in WLAN, ARF is thus not appropriate for MANETs whereby ensuring fairness among active nodes is an important requirement. The CARA scheme is a rate adaptation mechanism with abilities to distinguish between transmission failures due to channel errors and those due to collisions. The key idea behind CARA consists in the fact that a CARA sender decrements its rate after some consecutive transmission failures due to channel errors and increases its rate after some successful consecutive transmissions. In some cases, CARA largely improves the overall throughput, in comparison to the earlier-mentioned schemes. However, the CARA scheme does not take into account other competing nodes either, and therefore does not ensure system fairness. As we will show in the related work section, most, if not all, existing schemes do not jointly consider fairness, throughput efficiency, and transmission rate adaptation in MANET. Some consider only fairness; others consider only rate adaptation, while few methods consider both but in WLAN and not MANET. In [27], the authors propose airtime fairness in a rate separation IEEE 802.11b WLAN MAC. They group stations according to their transmission rates in different transmission periods. A superframe is decomposed into four parts corresponding to the four transmission rates. Each part is constituted of a beacon period and a data transmission period. An analytical formulation of the saturated throughput is presented. The simulation results show that the proposed rate separation gives advantages of airtime fairness and high saturation throughput. However, the introduction of super frame implies strong synchronization with the access point which coordinates and informs which stations, using the same data rate, can transmit in the following data transmission period. Moreover, this method cannot be applied in MANET, the network type REFOT is targeting.

\subsection{Contribution}

Classical REFOT (Relative Fairness and Optimized Throughput) is a new mechanism that increases the overall throughput via rate adaptation while maintaining fairness among nodes [2]. According to their access probability, nodes, competing for a particular channel, update their initial contention window size. Adjusting contention window and adapting the transmission rate shall enable nodes to have a certain fairness related to their perceived channel quality without compromising the system throughput.

In this paper, we propose an enhanced and extended version of our legacy REFOT mechanism [2]. The most significant added value consists in $i$ ) the modeling of REFOT using a 3-dimension Markov chain, ii) the introduction of an analytical performance evaluation that assists in the optimal tuning of the parameters of REFOT, iii) and the introduction of a new MAC algorithm considering all improvements following the analytical analysis. In REFOT, we assess the quality of a channel using information on transmission failures and successes. Based on this assessment of the channel quality, a tradeoff is to be retrieved between throughput and fairness. In general, nodes, competing for a particular channel, have access to the channel under different conditions, characterized by different parameters, such as nodes' mobility, nodes' density, traffic intensity, etc. In the modeling of REFOT, we take into account the channel quality which is evaluated by the transmitter before it selects its rate. Then, each node calculates its probability to access the channel while taking into account all its competing nodes. The transmitting node frequently updates this probability each time it desires to send data. REFOT allows for reaching the appropriate transmission rate level, without crossing all the intermediate levels. This operation helps in avoiding scenarios where the network capacity could be underutilized or overused, allowing the system to reach its stability faster.

We further optimize our model using an analytical evaluation and validate it using the Network Simulator (NS-2) [4]. In the performance evaluation, we consider different scenarios (i.e., by varying nodes' mobility, nodes' density and traffic intensity). The obtained results show that the new REFOT scheme outperforms its legacy counter part [2], the CARA scheme [17] and the classical DCF scheme (without any rate adaptation) [11].

\subsection{Organization}

The remainder of this paper is organized as follows. In Section II, we introduce the DCF mode of IEEE 802.11 and describe the RTS (Request-To-Send)/CTS (Clear-ToSend) mechanism. We also present existing rate adaptation schemes that are based on IEEE 802.11 standards. Section III describes our proposed analytical model. Section IV presents our proposed REFOT modeling using the three dimensions of Markov chain model. In Section VI, we show the simulation results and discuss them. Finally, Section VII concludes the paper.

\section{Related work}

\subsection{Preliminaries}

In this subsection, we briefly introduce the Distributed Coordination Function (DCF) of IEEE 802.11 standard and the RTS (Request-To-Send)/CTS (Clear-To-Send) mechanism. 


\subsubsection{IEEE 802.11 DCF}

The Distributed Coordination Function (DCF) mode combines Carrier Sense Multiple Access/Collision Avoidance (CSMA/CA) with the Request to Send/Clear to Send (RTS/CTS) handshake to avoid collisions [11]. When a node wants to transmit a packet, it first checks the channel status: if the medium is idle for a period of time longer than or equal to a Distributed Inter Frame Space (DIFS), the packet transmission begins in the following slot. Otherwise, the node should backoff for a certain period based on a value randomly selected from [0,CW], where $C W$ denotes the contention window size. The backoff value $C W$ is initially randomly selected from within the range $\left[0, C W_{\min }\right]$, where $\left(C W_{\min }=31\right)$. If the transmitted packet fails, due to collisions or Cyclic redundancy check (CRC) errors, $C W$ is doubled. $C W$ keeps on increasing until it reaches the upper bound CWmax, where $\left(C W_{\max }=2^{i_{\max }}-1\right)$, where $i_{\max }$ is the maximum retransmission number. When the transmission is successful, $C W$ is reset to $C W_{\min }$. Although DCF has a random backoff, it still cannot ensure collision-free transmissions, because it is possible that two or more nodes simultaneously finish the backoff. However, the collisions are not the only cause of transmission failures. Channel errors may also cause such failures. In this paper, we introduce the probability of channel access during the backoff time in order to reduce the number of transmission failures due to collisions or channel errors, and to ensure a relative fairness among the different competing nodes.

\subsubsection{RTS/CTS mechanism}

The original aim of the RTS/CTS mechanism is to reduce the impact of hidden nodes on the network. In fact, when hidden stations exist in the network, the performance of the basic CSMA/CA can be severely degraded. The unprotected time interval, however, can be shortened to the RTS transmission time, by preceding the data frame transmission with the exchange of two short control frames, i.e. RTS/CTS frames and hence the hidden station problem can be avoided. According to 802.11 standard [11], the decision to use the RTS/CTS exchange is made by only the transmitter. After a successful RTS/CST exchange, the channel is reserved for the next transmission.

In general, the RTS exchange is used when the size of the pending data frame is equal or larger than the RTS threshold value. However, in most 802.11 devices operating in WLANs environments with access points (APs), the RTS threshold is set to the maximum value (2347 octets) and is basically disabled in the real WLANs. RTS/CTS exchange is also used in heavily-contending WLAN environments, where many transmissions may fail due to collisions and the advantages could be amplified with a relatively large data frame. The RTS/CTS exchange is used to reserve a time interval, called transmission opportunity (TXOP) for consecutive transmissions of multiple data frames.

The main problem is that using RTS/CTS handshake before every data transmission wastes the time of data transmission, mainly when there is no such hidden terminal problem. Again this scheme does not take into account the channel quality and the effect of the presence of other competing nodes in MANETs and hence it may create unfairness among them. Moreover, the impact of fairness on Quality of Services (QoS) in IEEE 802.11 is presented by Bredel and Fidler in [7] and Berger et al. in [3]. Another work [5] focuses on fairness in MANETs with IEEE 802.11, but is only based on the backoff analysis without taking into account the rate adaptation [19].

\subsection{Rates adaptation schemes}

In this subsection, we will briefly introduce the existing rate adaptation mechanisms and discuss their limitations when applied in MANETs.

\subsubsection{Auto Rate Fallback (ARF)}

ARF developed for Lucent Technology' WaveLAN-2 WLAN device [13], has been widely implemented as a rate adaptation scheme because of its simplicity. In ARF, if two consecutive ACKs are not correctly received, then the next retrial of data transmission takes place at a lower rate and a timer is started. When a node receives 10 consecutive successful ACKs or when the timer expires, then the next transmission takes place at the next higher rate and the timer is set to zero. Indeed, ARF does not address the cause of transmission failures, i.e., channel errors or frame collisions. The advantage of this scheme is that it is easy to implement and is only based on timer and ACK packets. However, among the drawbacks of ARF, we can quote the throughput decrease, because a node decreases its rate even though transmission failures are caused by collisions. Furthermore, the scheme is not designed to ensure fairness between the competing nodes and is not adapted to MANETs characteristics.

\subsubsection{Time based Regulator (TBR)}

TBR is another solution proposed by Tan and Guttag [25] in order to provide long-term time-based fairness in AP-based WLANs. The main idea of TBR is based on temporal fairness and uses an algorithm to appropriately schedule packet transmissions. Other solutions based on time-based fairness are proposed in [26] [1]. In [1], Babu and Jacob introduced the idea of temporal fairness with DCF in multirate WLANs. In addition, the proposed mechanism is based on IEEE 802.11e standard [12] and MAC parameters have to be tuned properly to achieve temporal fairness. However, these solutions do not take into account the tradeoff between fairness and throughput, and are not suitable to MANETs, because they are proposed for the infrastructure mode WLAN. In this paper, we do not focus on the time-based fairness approach, because this kind of fairness is only suitable for a centralized network like WLAN which is not the case of MANETs. 


\subsubsection{Adaptive Multi-rate Auto Rate Fallback (AMARF)}

The AMARF scheme is proposed for IEEE 802.11 WLANs [28]. The key idea is to assign each data rate a unique success threshold, which is a criterion to switch one rate to the next higher rate, and the success thresholds can be dynamically changed in an adaptive manner according to the running conditions, such as packet length and channel parameters. Moreover, the AMARF protocol can be implemented without any change to the current IEEE 802.11 standards. The in-depth simulation shows that AMARF yields a significantly higher throughput than other existing schemes, including the ARF scheme and its variants, under various running conditions. However, although AMARF outperforms the ARF scheme, it does not take into account the competing nodes in MANETs context, and then the fairness parameter is not considered.

\subsubsection{Cross Layer Rate Adaptation (CLRA)}

The basic idea behind the CLRA scheme [16] is that the rate adaptation mechanism should select a data rate according to the channel state variations and application requirements. The mechanism needs a cross layer framework to be implemented. The channel conditions are constantly monitored using a statistical approach based on the number of frame losses as a measure to identify the channel state. However, loss differentiation is used in order to make intelligent decisions regarding bad channel conditions. The transmitter selects such a data rate value so that the amount of outgoing traffic is equal to the traffic which can effectively be transmitted by the transmitter within a particular time under given MAC sub-layer timing constraints. Consequently, CLRA mechanism performs better than the other rate adaptation mechanisms. However, the limitation of CLRA consists in the fact that it is not applicable to MANETs and does not ensure fairness.

\subsubsection{Collision-Aware Rate Adaptation (CARA)}

CARA uses the RTS/CTS mechanism to estimate the quality of the channel [17]. Unlike ARF, CARA distinguishes between collisions and channel errors when a transmission fails. Depending on the cause of the transmission failure, a transmitter node decrements its rate only in case of consecutive channel errors, but not in case of collisions. The RTS/CTS mechanism is enabled only when the number of transmission failures reaches a certain degree. That is why CARA uses the RTS probing to decide to enable or disable RTS/CTS exchanges. Furthermore, CARA improves the throughout and gives better results than ARF and the classical RTS/CTS mechanism in many cases. It is able to differentiate collisions from channel errors at the transmitter side without any help/feedback from the receiver station. In addition, with CARA, unnecessary rate shrinkage can be avoided. However, CARA scheme is well adapted for WLANs, yet not suitable for MANETs and does not support system fairness.
Other existing works in literature dealt with auto adaptation rate. In [23], the Opportunistic Auto Rate (OAR) protocol is proposed for multirate in MANET in order to ensure that nodes with good channel conditions access the channel for a long duration. However, OAR is based on a DCF protocol and represents an extension to previous proposed rate adaptation schemes $[15,10]$. The main drawback of OAR is related to its implementation, which requires complete modifications to DCF. Another study focuses on the effects of contention unfairness in CSMA networks based on IEEE 802.11 particularly in Wireless Multi-hop Networks [18]. However, this study is not adaptable to MANETs context, because some parameters like the mobility impact are not taken into account.

We distinguish four main criteria to classify fairness mechanisms: time fairness, throughput fairness, max-min fairness, and proportional fairness. The aim of the time fairness and throughput fairness mechanisms is to equally distribute the resources to stations in terms of channel occupation time and throughput [14]. Usually these mechanisms try to reach the absolute fairness between competing nodes. However, max-min fairness and proportional fairness are defined as optimization problems. In this paper, we used relative fairness in order to differentiate compared to absolute fairness where the competing nodes must have the same times to access the channel and for the same time duration. It is very challenging to guarantee an absolute fairness in MANETs because of their characteristics. For example the channel conditions are not the same for competing nodes. That is why, we use the relative fairness as terminology. Hence, the trade-off between fairness and throughput is studied in order to ensure relative fairness with the optimization of throughput.

\section{REFOT: Relative Fairness And Optimized Throughput}

The REFOT scheme is proposed for IEEE 802.11 with DCF mode in the context of MANETs. The goal of the REFOT scheme is to ensure relative fairness among competing nodes without compromising the throughput. The key idea is based on the channel quality and on the assessment of transmission failures and transmission successes. In addition, the probability to access a channel is introduced in the BEB (Binary Exponential Backoff) mechanism while taking into account the set of competing nodes. Unlike CARA, REFOT allows rate adaptation as well as relative fairness with an optimal throughput. The set of notations used in this paper is listed in table 1.

Before a node selects its rate for the data transmission, it assesses the number of its consecutive failure transmissions $(n)$ and the number of consecutive successful transmissions $(s)$. A successful transmission is validated with the reception of the ACK frame. However, when the number of consecutive transmission failures reaches a certain threshold value $P_{t h}$, the RTS/CTS mechanism is activated for the next data transmission. Thus the 
Table 1: Table of Variables and Notation

\begin{tabular}{|l|l|}
\hline \hline$n$ & Number of consecutive transmission failures \\
$s$ & Number of consecutive successful transmissions \\
$P_{t h}$ & Threshold of transmission failures needed to ac- \\
& tivate the RTS/CTS mechanism \\
$N_{t h}$ & $\begin{array}{l}\text { Threshold of transmission failures needed to de- } \\
\text { crease the rate }\end{array}$ \\
$M_{t h}$ & $\begin{array}{l}\text { Threshold of successful transmissions needed to } \\
\text { increase the rate }\end{array}$ \\
$\Phi_{J}$ & Set of nodes competing for Channel J \\
$R_{-} d t$ & Set of available rates \\
$K$ & Total number of transmissions \\
$f$ & Total number of transmission failures \\
$P_{d}^{J}$ & Probability that node d fails to transmit through \\
$Q_{d}^{J}$ & channel J \\
$F I$ & Probability that node d accesses channel J \\
$Z$ & Fairness Index \\
\hline
\end{tabular}

RTS/CTS mechanism is efficiently used. Unlike Classical REFOT, we distinguish two cases for decreasing the transmission rate. If the current rate is the lowest, when $n$ the number of consecutive transmission failures reaches $m\left(m \geq N_{t h}\right)$, the next attempts for transmissions continue with the same lowest rate. However, in higher transmission rates, when $n$ reaches $N_{t h}$ the transmitting node decreases its rate by selecting the lower rate from the set $\left(R_{-} d t\right)$ and resets counter $n$. In the simulations, we will consider the set of rates available in IEEE 802.11b which is $\{1,2,5.5,11\} M b p s$. If the number of consecutive successful transmissions $(s)$ reaches a certain threshold number $M_{t h}$, the transmitting node selects the next higher rate in set $R_{-} d t$. Then, the transmitter computes its probability to access the channel and adapts the size of the backoff window. According to the value of the backoff window, the transmitter will choose a new transmission stage which is presented in the next section.

In order to define the probability to access a channel, we introduce two variables: the total number of transmissions $K$ and the total number of transmission failures $f$ till the current time $t$. A channel is defined as the radio coverage area shared by competing nodes of the sender. Let's consider a node d which belongs to a channel $J$. The probability of any transmission failure for node $d$ is:

$$
P_{d}=\frac{f_{d}}{K_{d}}
$$

According to the rate adaptation mechanism, the current transmission rate $r$ is known. We introduce a new probability that is a metric to measure the channel quality. If a packet is transmitted through a Channel $J$, then the probability that a transmission fails for node $d$ is given by:

$$
P_{d}^{J}=\frac{\sum_{l \in \Phi_{J}} P_{l} r_{l}}{\sum_{l \in \Phi_{J}} r_{l}}
$$

where $\Phi_{J}$ is the set of nodes competing for channel $\mathrm{J}, r_{l}$ is the transmission rate of Node $1, P_{l}$ is the probability of failure of node 1 . This probability takes into account different reasons of transmission failure(i.e., collision, error). This equation shows how node $d$ computes the failure probability to access channel J. It is important to take into account not only the probability of failure of competing nodes $\left(\in \Phi_{j}\right)$, but also their rates $r_{l}$ used to access channel J. The rate selected by the competing nodes directly impacts the failure probability to access the channel. Hence, each node $d$ must inform its neighbors about these values $r_{d}$ and $P_{d}$. For this purpose, the four address fields ( 6 bytes) in the MAC header can be used since they are not needed in the ad-hoc mode. We can also use the management packets provided by the MAC layer to send this information. At the reception of such information, each node calculates the probability to access the main channel:

$$
Q_{d}^{J}=\frac{1-P_{d}^{J}}{\sum_{l \in \Phi_{J}} 1-P_{l}^{J}}
$$

where $\Phi_{J}$ is the set of nodes competing for channel J. This probability takes into account the channel conditions of the neighboring nodes. According to this probability, each node respects the other neighbor nodes by introducing their probability to access the communication channel. Then, this probability is incorporated to fix the value $C W_{\min }^{*}$ of the transmitting node as follows:

$$
C W_{\text {min }}^{*}= \begin{cases}C W_{\min } \cdot\left(1-Q_{d}^{J}\right) & \text { if } Q_{d}^{J} \neq 1 \\ C W_{\min } & \text { Otherwise }\end{cases}
$$

When a node connects to a channel, it does not have prior knowledge on the presence of other competing nodes. In that case, we assume that $Q_{d}^{J}=1$. This assumption prevents the new comers from accessing the channel, we let them go backoff without affecting $C W_{\min }$. Thus, the backoff of all competing nodes is affected and these nodes access the channel based on their probability to access the channel. This yields relative fairness among the nodes based on their channel quality to access the channel before adapting their transmission rates, respectively.

\section{Markov Chain Model For REFOT}

In this section, we present the details of our throughput model proposed for REFOT scheme. In our model, we made the same assumptions as in [6]. First, we present the analysis of the DCF mode. Second, we develop the mathematical model for REFOT. Both fairness and throughput are taken into account in the models. We first study $\tau$, which is the transmission probability of a single station during a randomly selected time slot. Then, we express the throughput model for the whole network as a function of the variable $\tau$. 


\subsection{Analysis of the DCF mode}

We briefly present the existing model developed for the DCF mode [6]. Let $b(x)$ be the stochastic process that represents the backoff time counter for a given station and $s(x)$ be the stochastic process that represents the backoff stage. Let $W_{i}$ be the contention window size, which is defined as $W_{i}=2^{i} W_{0}$, where $W_{0}=C W_{\text {min }}$ is the initial contention window value. Let $W_{m}=C W_{\max }$ be the maximum value of the contention window where $m$ is the maximum backoff stage. We denote by $\mathrm{p}$ the conditional collision probability, which is constant and independent regardless of the number of retransmissions incurred. Therefore, the system can be modeled as a 2-D stochastic process $\{s(x), b(x)\}$. We can now express the probability $\tau$ that a station transmits in a randomly chosen slot time:

$$
\tau=\frac{2(1-2 p)}{(1-2 p)\left(W_{0}+1\right)+p W_{0}\left[1-(2 p)^{m}\right]}
$$

\subsection{Analysis of REFOT scheme}

In REFOT the transmitter adjusts its backoff $\left(C W_{i, j}\right)$ according to the probability to access the channel $\left(Q_{d}^{J}\right)$ defined by Equation 3. However, for modeling reasons, we define four classes of backoff which represent the different rates used by the transmitter. The contention window size $\left(W_{i, j}\right)$ is then defined as:

$$
W_{i, j}=\alpha_{j} 2^{i} W_{0},
$$

where $\alpha_{j} \in\{1,3 / 4,2 / 4,1 / 4\}, j \in[0,3]$ the backoff stage, $i \in[0, m]$ the counter of successful and failed transmissions and $W_{0}=C W_{\text {min }}$. Recall that in REFOT, a station increases its rate after $M$ consecutive successful transmissions. However, it decreases its rate after $N$ consecutive failure transmissions and its actual transmission rate is not the lowest one $(j>0)$. In the case of the lowest rate $(j=0)$, REFOT uses the threshold $m$ as maximum retransmission attempt like in standard IEEE 802.11. When the rate is increased, the maximum retransmission threshold is set to $N$ where $N \leq m$. REFOT reduces the maximum number of transmission attempts to prevent the burst of transmission failures from occurring. However, it increases this number when the rate is the lowest. Accordingly, we obtain a $3-D$ stochastic process $\{u(t), v(t), w(t)\}$ to model REFOT scheme as a discrete-time Markov chain as illustrated in Fig. 1. The first process $u(t)$ represents the current backoff stage $(j \in[0,3])$. The second process $v(t)$ represents the number of consecutive successful and failure transmissions experienced by a station which is explained hereafter:

- $v(t)=-i, i \geq 1$ represents a station that has suffered i consecutive failure transmissions.

- $v(t)=i, i \geq 1$ represents a station that has experienced i consecutive successful transmissions.

- $v(t)=0$, indicates the first step of each rate $(j)$.
The third process $w(t)$ represents the backoff counter for a given station. A discrete and integer time scale is adopted as in Bianchi model [6]. According to the Markov chain illustrated in Fig. 1, the non-null one-step transitions are given by:

$$
\left\{\begin{array}{l}
P(j, i, k-1 \mid j, i, k)=1 \\
P(j, i-1, k \mid j, i, 0)=\frac{p}{W_{i+1, j}} \\
P(j, i+1, k \mid j, i, 0)=\frac{(1-p)}{W_{0, j}} \\
P(j,-1, k \mid j, i, 0)=\frac{p}{W_{1, j}} \\
P(0,-m, k \mid 0,-m, 0)=\frac{p}{W_{m, 0}} \\
P(r, 0, k \mid j, M, 0)=\frac{(1-p) \alpha_{r}}{W_{0, r}} \\
P(j, 1, k \mid j,-i, 0)=\frac{(1-p)}{W_{0, j}} \\
P(r, 0, k \mid j,-N, 0)=\frac{p \alpha_{r}}{W_{0, r}}
\end{array}\right.
$$

where $\alpha_{r} \approx Q_{d}^{J}$ if $\alpha_{r}-1 / 4<Q_{d}^{J} \leq \alpha_{r}$ and $\alpha_{r} \in$ $\{1,3 / 4,2 / 4,1 / 4\}$. In addition, the corresponding ranges for $\mathrm{i}, \mathrm{j}$ and $\mathrm{k}$ are given by:

$$
\left\{\begin{array}{l}
i \in[-m, M], j \in[0,3], k \in\left[0, W_{i, j}-1\right] \\
i \geq 0, j \in[0,3], k \in\left[0, W_{i+1, j}-1\right] \\
M>i \geq 0, j \in[0,3], k \in\left[0, W_{0, j}-1\right] \\
i \geq 0, j \in[0,3], k \in\left[0, W_{1, j}-1\right] \\
k \in\left[0, W_{m, 0}-1\right] \\
j \in[0,3], 3 \geq r>j, k \in\left[0, W_{0, r}-1\right] \\
j \in[0,3], k \in\left[0, W_{i, j-1}-1\right] \\
j \in[1,3], j>r \geq 0, k \in\left[0, W_{i, r}-1\right]
\end{array}\right.
$$

The set of transition probabilities defined in (6) are explained hereafter:

- $P_{(j, i, k+1) \rightarrow(j, i, k)}$ : A station will decrease its backoff counter by 1 after it senses that the channel is idle for each time slot; otherwise, it will freeze its backoff.

- $P_{(j, i, 0) \rightarrow(j, i-1, k)}$ : If the station fails i consecutive transmissions where $\mathrm{i}$ is less than the maximum retransmission (if $\mathrm{j}=0$ then, $i<-m$ - if $j>0$ then $i<-N)$, it will increase its backoff $\left(W_{i, j} \rightarrow W_{i+1, j}\right)$ and decrement counter $i$.

- $P_{(j, i, 0) \rightarrow(j, i+1, k)}$ : The station increases its counter $i$ after each successful transmission if $i<M$.

- $P_{(j, i, 0) \rightarrow(j,-1, k)}$ : When the station fails its transmission after $i$ successful transmissions, it will increase its backoff and set $i$ to -1 .

- $P_{(0,-m, 0) \rightarrow(0,-m, k)}$ : When the station fails its transmission after $m$ times in case of the lowest rate $(j=0)$, it will keep the same upper bound $W_{m, 0}$ until it achieves a successful transmission.

- $P_{(j, M, 0) \rightarrow(r, 0, k)}:$ When the station succeeds in transmitting $M$ consecutive packets, then the next transmission will occur with a new contention window $W_{0, r}$ 


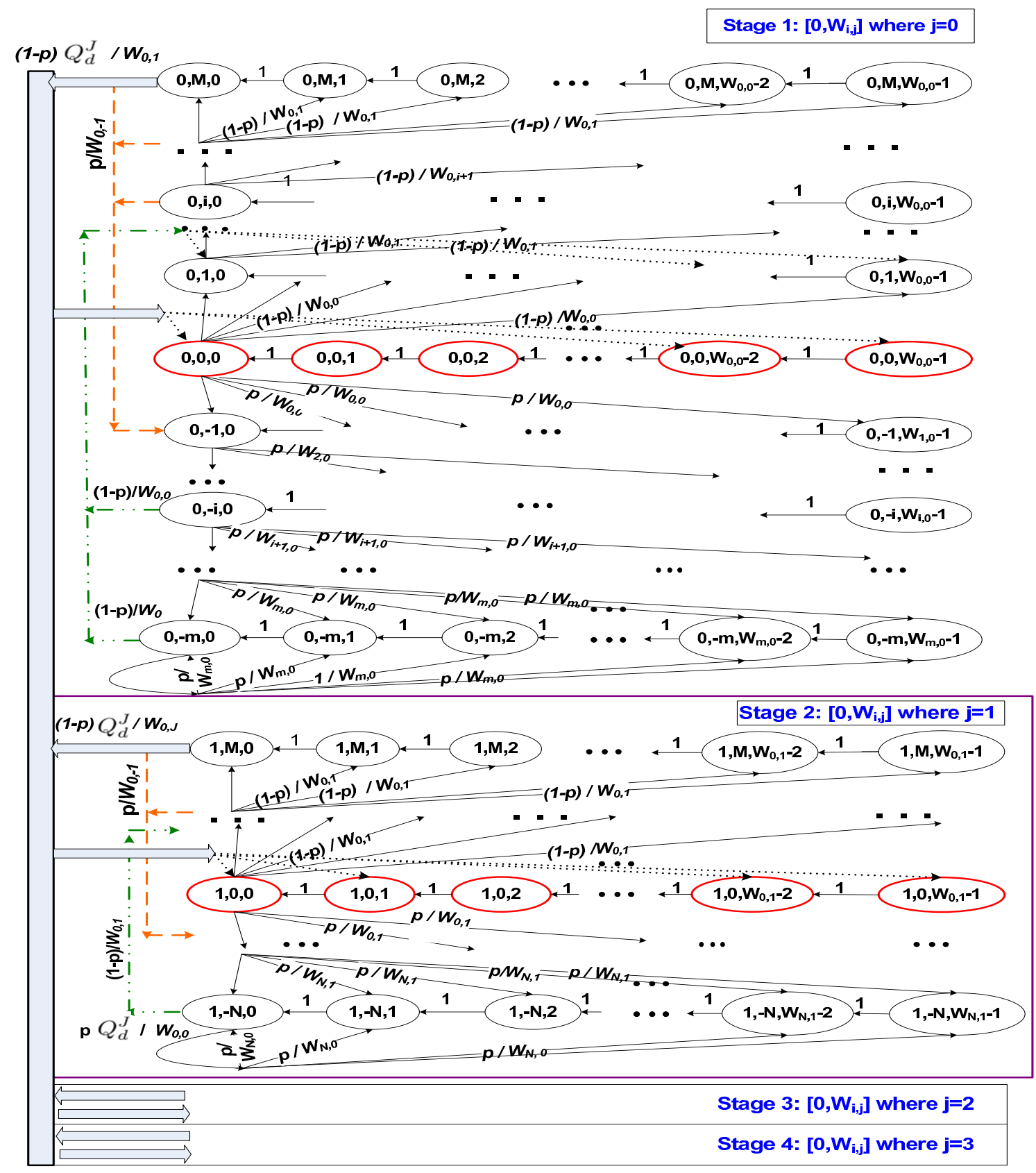

Figure 1: Markov chain model (3-D) for REFOT. 
calculated according to $Q_{d}^{J}$. If $0 \leq j<3$ then the station will increase its rate according to $Q_{d}^{J}$. However, if $j=3$, then the actual rate used by the transmitter is the highest one, and it keeps its current rate.

- $P_{(j, i, 0) \rightarrow(j, 0, k)}$ : Once the station failed to transmit $i$ packets, then it will update its contention window from $W_{i, j}$ to $W_{0, j}$ without changing its rate.

- $P_{(j,-N, 0) \rightarrow(r, 0, k)}$ : This probability represents the case of $\mathrm{N}$ transmission failures that happened at the transmitter station. The station then decreases its rate according to $Q_{d}^{J}$ if $j>0$.

As illustrated in Fig. 1, the station d selects its stage from the four available stages according to probability $Q_{d}^{J}$. Once the stage is selected, the station starts the transmission with $i=0$. In case the transmission succeeds, the station keeps the same upper bound of backoff $W_{0, j}$ and increments the successful transmission counter $(v(t))$. If the station transmits $M$ consecutive successful transmissions, then it increases its rate according to probability $Q_{d}^{J}$. While $v(t)<M$, the station stays in the same stage j. However, if the transmission fails, the station will increase the upper bound size (backoff window) and it sets the counter $v(t)$ to -1 , and then retransmits the packet. The station decreases its rate based on the probability $Q_{d}^{J}$ when the consecutive transmission failures reach $N$. When $(v(t) \neq N)$, then the station uses the classical backoff algorithm and continues to decrement the counter $v(t)$. However, when $(j=0)$, that means that the rate used is the lowest one and that the threshold of maximum retransmission attempts is $m$ where $m \geq N$ and $N$ is used when the used rate is not the lowest one.

Let $b_{j, i, k}=\lim _{t \rightarrow \infty} P\{u(t)=(i, j), v(t)=\gamma, w(t)=$ $k\}$, where $i \in[0, m], j \in[0,3], \gamma \in[-m, M]$ and $k \in$ $\left[0, W_{i, j}\right]$ be the stationary distribution of the chain. For the solution of this Markov chain, we suppose that the probability of collision $\mathrm{p}$ for node $\mathrm{d}$ is calculated by $P_{d}$ (see Equation 1), then $p=P_{d}$. We take into account the two following cases in our study: 1$)$ the case of $(j=0)$ and 2) the case of $0<j \leq 3$.

a) Case of $j=0$ : In this case, the station uses the lowest rate, and if a transmission fails, a station will double its upper bound until it reaches $W_{m, 0}$. However, the number of consecutive successful transmissions does not change the upper bound $W_{0,0}$ until it reaches the maximum threshold M. In order to calculate $b_{0, i, k}$, we distinguish two cases depending on value $i$.

When $i<0$, then $b_{0, i, k}$ is calculated as follows:$$
b_{0,-i, k}=\frac{W_{-i, 0}-k}{W_{-i, 0}}\left\{\begin{array}{l}
p^{-(i+1)} b_{0,0,0} \quad \text { if }-m<i<-1 \\
p\left(b_{0,-(m-1), 0}+b_{0,-m, 0}\right) \quad \text { if } i=-m \\
p \sum_{r=1}^{M} b_{0, r, 0} \quad \text { if } i=-1
\end{array} \quad \tau=\sum_{j=0}^{3} \sum_{i=-m}^{M} b_{j, i, 0}\right.
$$

When $i \geq 0, b_{0, i, k}$ is calculated as follows:

$$
b_{0, i, k}=\frac{W_{0,0}-k}{W_{0,0}} \begin{cases}p \alpha_{0} \sum_{j=1}^{3} b_{j,-N, 0} & \text { if } \mathrm{i}=0 \\ (1-p) \sum_{r=1}^{m} b_{0,-r, 0} & \text { if } \mathrm{i}=1 \\ (1-p)^{i-1} b_{0,0,0} & \text { if } 1<i \leq M\end{cases}
$$

b) Case of $0<j \leq 3$ : In this case, the station d does not use the lowest rate. When the transmission fails, the station doubles its upper bound until it reaches $W_{N, j}$ where $N$ represents the maximum number of consecutive retransmission failures, before the station changes its rate to the lowest one according to probability $Q_{d}^{J}$. However, the maximum number of consecutive transmissions is $M$. Then, when this value is reached the station will increase its rate according to probability $Q_{d}^{J}$. In order to calculate $b_{0, i, k}$, we distinguish two cases depending on the value of $i$. When $i<0$, then $b_{j, i, k}$ is calculated as follows:

$$
b_{j,-i, k}=\frac{W_{-i, j}-k}{W_{-i, j}} \begin{cases}p^{-(i+1)} b_{j, 0,0} & \text { if }-N \leq i<-1 \\ p \sum_{r=1}^{M} b_{j, r, 0} & \text { if } \mathrm{i}=-1\end{cases}
$$

When $i \geq 0$, then $b_{j, i, k}$ is calculated as follows:

$b_{j, i, k}=\frac{W_{0, j}-k}{W_{0, j}}\left\{\begin{array}{l}(1-p)\left(\sum_{r=1}^{N} b_{j,-r, 0}+b_{j, 0,0}\right) \quad \text { if } \mathrm{i}=1 \\ (1-p)^{i-1} b_{j, 0,0} \quad \text { if } 1<i \leq M \\ \alpha_{j}\left(p \sum_{r=1}^{3} b_{r,-N, 0}+(1-p) \sum_{s=0}^{2} b_{s, M, 0}\right) \text { if } i=0\end{array}\right.$

if $i=0$, parameters $\mathrm{r}, \mathrm{j}$ and $\mathrm{s}$ meet these conditions: $r>j$ and $s<j$. We need to calculate $b_{0,0,0}$ to resolve the following equation.

$$
1=\sum_{j=0}^{3} \sum_{i=-m}^{-1} \sum_{k=0}^{W_{-i, j}-1} b_{j,-i, k}+\sum_{j=0}^{3} \sum_{i=0}^{M} \sum_{k=0}^{W_{0, j}-1} b_{j, i, k}
$$

The different relation between $b_{j, 0,0}$ where $j \in\{0,1,2,3\}$ is given by the following system of equations:

$$
\left\{\begin{array}{l}
b_{0,0,0}=\alpha_{0} p^{N} \sum_{i=1}^{3} b_{i, 0,0} \\
b_{1,0,0}=\alpha_{1}\left((1-p)^{M} b_{0,0,0}+p^{N} \sum_{i=2}^{3} b_{i, 0,0}\right) \\
b_{2,0,0}=\alpha_{2}\left((1-p)^{M} \sum_{i=0}^{1}+p^{N} b_{3,0,0}\right) \\
b_{3,0,0}=\alpha_{3}(1-p)^{M} \sum_{i=0}^{2} b_{i, 0,0}
\end{array}\right.
$$

Therefore, we can write $b_{j, 0,0}$ where $\mathrm{j} \in\{1,2,3\}$ according to $b_{0,0,0}$.

Given the fact that any transmission occurs when the backoff time counter is equal to zero, the probability $\tau$ can expressed as follows:

$$
\tau=\sum_{j=0}^{3} \sum_{i=-m}^{M} b_{j, i, 0}
$$




\section{Single station transmission probability}

Once the backoff time counter reaches 0 , a transmission will start regardless of the backoff stage:

$$
\begin{gathered}
\tau=\sum_{j=0}^{3} \sum_{i=-m}^{M} b_{j, i, 0}=\frac{1}{1-p} \sum_{j=0}^{3} b_{j, 0,0}+\frac{1}{p} \sum_{j=0}^{3} b_{j, 0,0} \\
\tau=\sum_{j=0}^{3} \frac{1}{p(1-p)} b_{j, 0,0}
\end{gathered}
$$

From Eq. 9, we can see that $\tau$ is a function of only one unknown variable $p$, as $b_{j, 0,0}$ can be numerically solved. Assuming that the average number of neighbors is $n$, then, the probability that a collision occurs is calculated as follows:

$$
p=1-(1-\tau)^{Z-1}
$$

where $Z$ denotes the total number of nodes in the given topology.

Eq. 9 and 10 represent a non linear system with two unknown variables $\tau$ and p. It can be proved that this system has a unique solution, because the probability of collisions $p$ is a continuous and a monotone function which increases with $\tau$. Probability $\tau$ is proved by the fact that the first order derivation of $\mathrm{p}$ is always larger than zero:

$$
p^{(1)}=(Z-1)(1-\tau)^{Z-2}
$$

\section{Total Network Throughput Model}

Here we present the total network throughput model for REFOT. Let $P_{t r}$ be the probability that there is at least one transmission in a considered time slot:

$$
P_{t r}=1-(1-\tau)^{Z}
$$

Let $P_{s}$ be the probability that a transmission is successful. Intuitively, this is under the condition that only one station transmits on the channel:

$$
P_{s}=\frac{n \tau(1-\tau)^{Z-1}}{P_{t r}}=\frac{Z \tau(1-\tau)^{Z-1}}{1-(1-\tau)^{Z}}
$$

Hence the expression of the normalized system throughput is given by:

$$
\Gamma=\frac{P_{s} P_{t r} E[P L]}{\left(1-P_{t r}\right) T_{0}+P_{t r} P_{s} T_{s}+P_{t r}\left(1-P_{s}\right) T_{f}}
$$

where:

- $E[P L]:$ average packet payload size

- $T_{0}$ : duration of an empty slot time

- $T_{s}$ : for which a channel is sensed busy for a successful transmission

- $T_{f}$ : average time for which a channel is sensed busy for a failed transmission

In [6], the mathematical expressions of $E[P], T_{0}, T_{s}$ and $T_{f}$ are provided. Due to space limitations, we are not providing them here.

\subsection{REFOT algorithm}

Figure 2 summarizes the REFOT flowchart. When an MAC Protocol Data Unit (MPDU) is ready to be sent, a node executes the following MAC algorithm. At the beginning, the access probability $Q_{d}^{J}$ is equal to 1 and the parameters $\{n, s, K, f\}$ are set to zero. The first step consists in parameters initialization with RTS/CTS mechanism disabled. The node continuously refreshes the set of parameters $\left\{P_{l}, r_{l}\right\}$ received by overhearing the transmission of neighboring nodes. Then, it calculates the probability to access the channel $\left(Q_{d}^{J}\right)$. If this probability is equal to 1 the backoff algorithm is not changed. Otherwise, the node changes the backoff algorithm (BEB) parameters by introducing the new $C W_{m i n}^{*}$. Once the node finishes this step, it adds its own refreshed parameters in terms of probability and transmission rate in the scheduled packet. In the second step, the node checks the packet transmission. In the case of a transmission failure the counter parameter $n$ is incremented and the counter $s$ is set to zero. According to the threshold $N_{t h}$ the decision to decrease the transmission rate is made as illustrated in the flowchart. However, in the case of a transmission success the counter parameters $s$ and $n$ are updated and according to the thresholds $M_{t h}$ the transmission rate is increased or not. Moreover, the decision to enable the RTS/CTS mechanism is made according to the threshold $P_{t h}$ and $R T S_{T h} . R T S_{T h}$ indicates the threshold of packet size. When size of MPDU exceeds the $R T S_{T h}$, the RTS/CTS mechanism is enabled to prevent the collision and the hidden nodes problem. The recommended $R T S_{T h}$ value is set to 500bytes.

The difference between classical REFOT and REFOT is in the way to increase and decrease the current rate (dashed boxes in Fig. 2). In the case of classical REFOT, the rate is increased gradually without using the probability $Q_{d}^{J}$ which means that the node d cannot increase directly its rate from $1 M$ to $5,5 M$ without passing by $2 M$. However, in the case of REFOT, the rate is dynamically increased according to the following probability $(1-p) Q_{d}^{J} / W_{0, j}$ computed from Markov chain model; which means the node can directly increase its rate from $1 M$ to $5,5 M$. In the next section we evaluate both REFOT versions with and without Markov chain model.

\section{Numerical Analysis}

In this section, we first analytically evaluate our proposed model. Table 2 summarizes the considered parameters.

In order to show the impact of the probability of collision $(p)$ on the probability of transmission $(\tau)$, we plot in Fig. 3 , the probability $\tau$ for different values of the probability of collision $(p)$ considering different values of $N$ (threshold of consecutive transmission failures) and $M$ (threshold of consecutive successful transmissions). We notice that when the probability of collision increases the probability of transmission decreases in both cases of REFOT and 


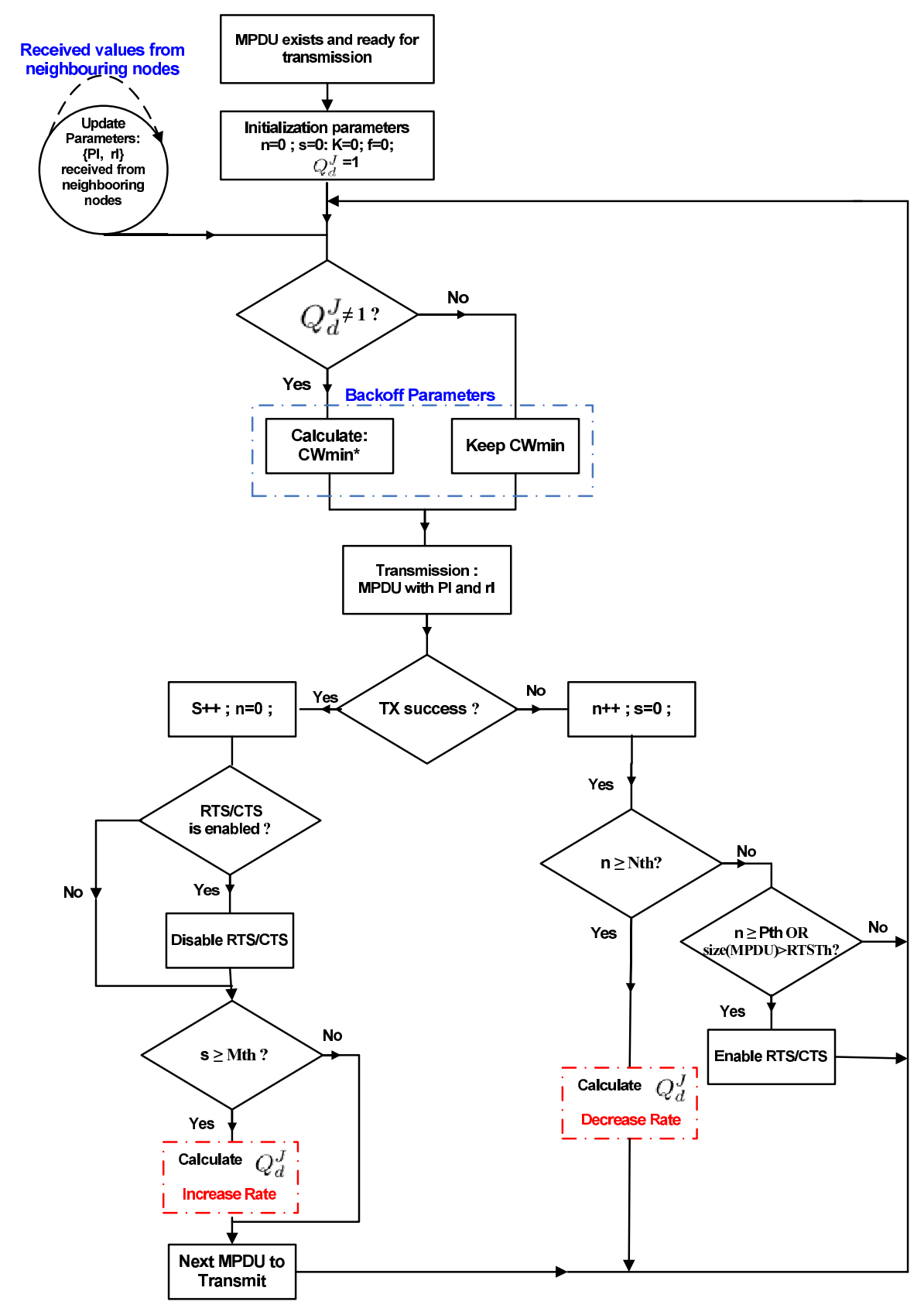

Figure 2: REFOT Flow-chart. 
Table 2: Parameters for Analytical Results

\begin{tabular}{|l|l|}
\hline Parameters & Value \\
\hline \hline Payload $E(P L)$ & 1050 Bytes \\
Physical Header & 28 Bytes \\
MAC Header & 52 Bytes \\
RTS & 44 Bytes \\
CTS & 38 Bytes \\
ACK & 38 Bytes \\
\hline SIFS & $10 \mu s e c$ \\
DISF & $50 \mu s e c$ \\
Slot Time & $20 \mu s e c$ \\
Propagation Delay & $2 \mu s e c$ \\
\hline$C W_{\min }$ & 31 \\
$C W_{\max }$ & 1023 \\
\hline
\end{tabular}

standard DCF. However, in case of REFOT the probability of transmission is higher than in the case of DCF. Moreover, the impact of parameters $N$ and $M$ is more important when the probability of collision is small (ie. less than 0.5 ) and is minimal when probability $p$ is higher than 0.5 .

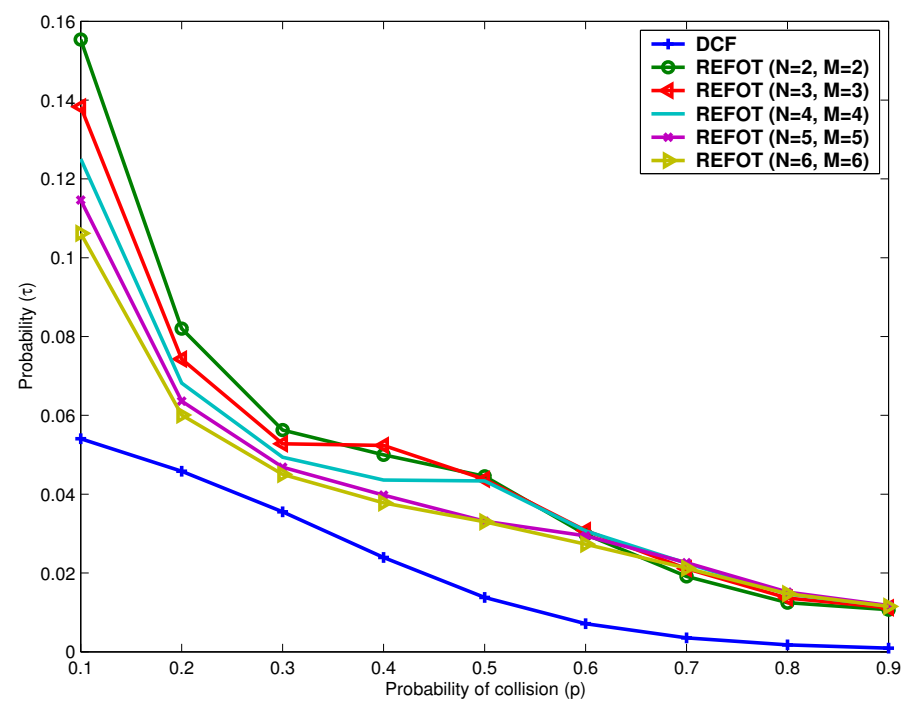

Figure 3: Transmission probability $(\tau)$ versus probability of collision $(p)$.

The throughput is an important metric to evaluate our proposed model for REFOT. That is why we plot in Fig. 4 the normalized throughput for different numbers of nodes $(Z)$. We notice that the throughput decreases when the number of nodes increases, due to the high competition between nodes to access the channel. In addition, when we vary parameters $N$ and $M$ with the same values, we remark that there is no significant change in the throughput. However, we focus on these parameters $N$ and $M$ to analyse the heterogenous and different values. In Fig. 5(a) and $5(\mathrm{~b})$, the normalized throughput is plotted for different values of $\mathrm{N}$ and $\mathrm{M}$. The obtained results in the case of a low density of nodes $(Z=5)$ are plotted in Fig. 5(a). We notice that the impact of parameter $M$ is insignificant, mainly due to the high probability of transmission in the case of a low density of nodes. In addition, parameter $M$ represents the threshold of consecutive successful transmissions, which indicates the successful transmissions of a node. We know that when the density of nodes is low, the probability of collision becomes also low. Therefore, as Fig. 3 shows, when the probability of collision is low, the transmission probability is higher. However, the threshold of consecutive transmission failures $(N)$ has an important impact on the throughput, as a node stays for a long time in the same stage before decreasing and changing its rate. In other words, when $N$ is high, a node may take a long time when the conditions to access a channel are bad before decreasing the rate in order to reduce the transmission failures. In the case of a high density of nodes, we plot the

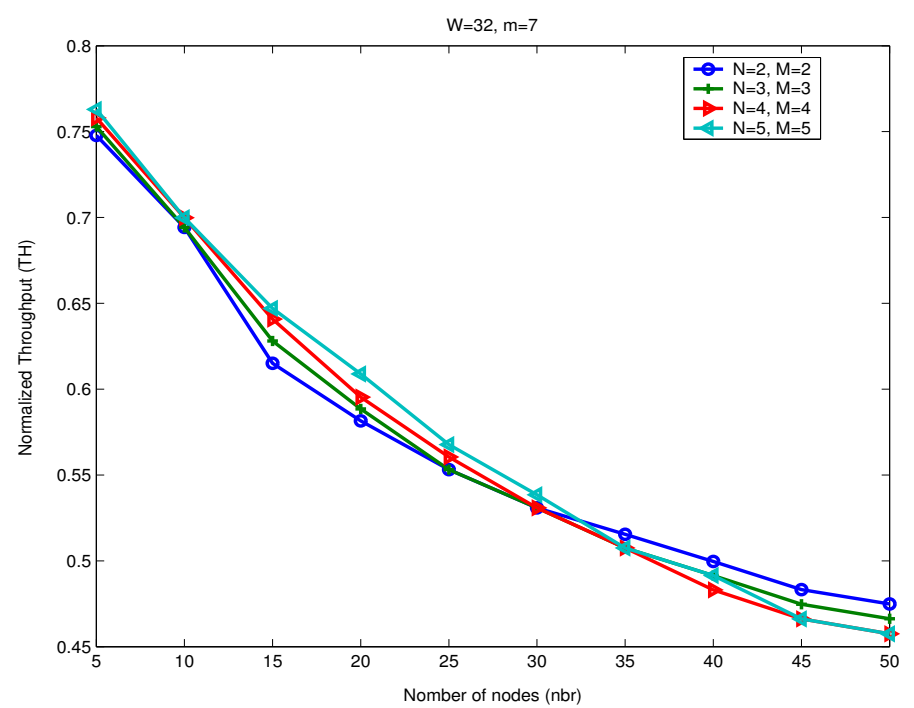

Figure 4: Normalized throughput versus number of nodes.

obtained results (with 20 as number of nodes) in Fig. 5(b). We notice that the normalized throughput is high when both parameters $M$ and $N$ are high. In other words, the stage of rates get stable values with high values of parameters $M$ and $N$ when the density of nodes is important. We deduce that parameter $M$ has a more significant impact than in the previous scenario and that is mainly due to the fact that the probability of successful transmissions is low in this case. Recall that $M$ is the threshold of consecutive successful transmissions. Then when $M$ is high, a node increases its rate less frequently, which is important in case of bad channel conditions.

As a conclusion to this study, we can say that the throughput depends on the rate level which depends on parameters $N$ and $M$. Moreover, the choice of these parameters is highly important and difficult at the same time, because they depend on other parameters, such as the density of nodes, the quality of channel, etc. In order to get good throughput results,parameters $N$ and $M$ must be 


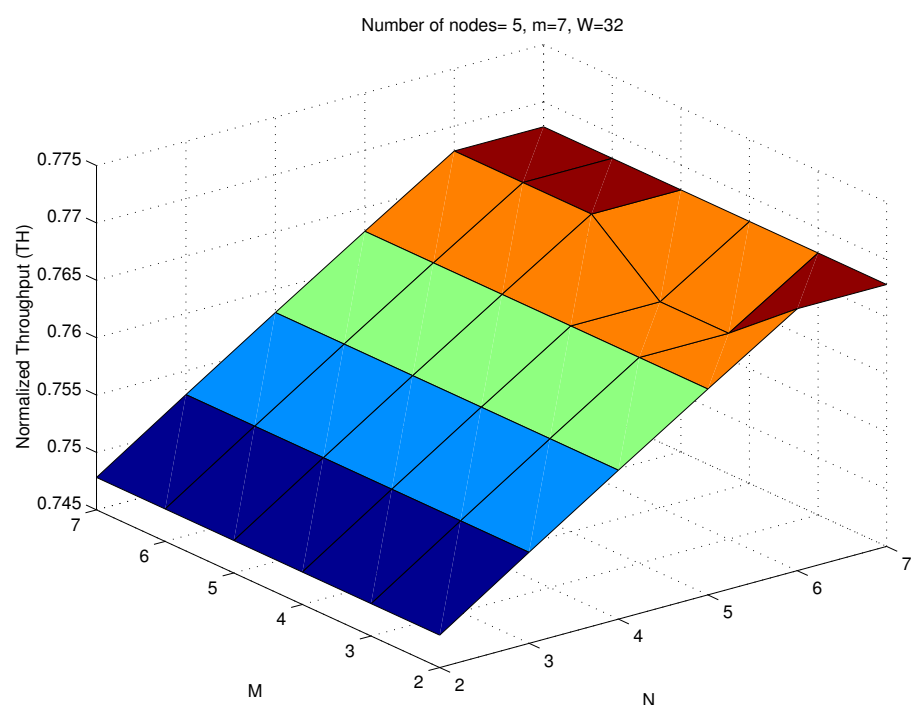

(a) 5 nodes

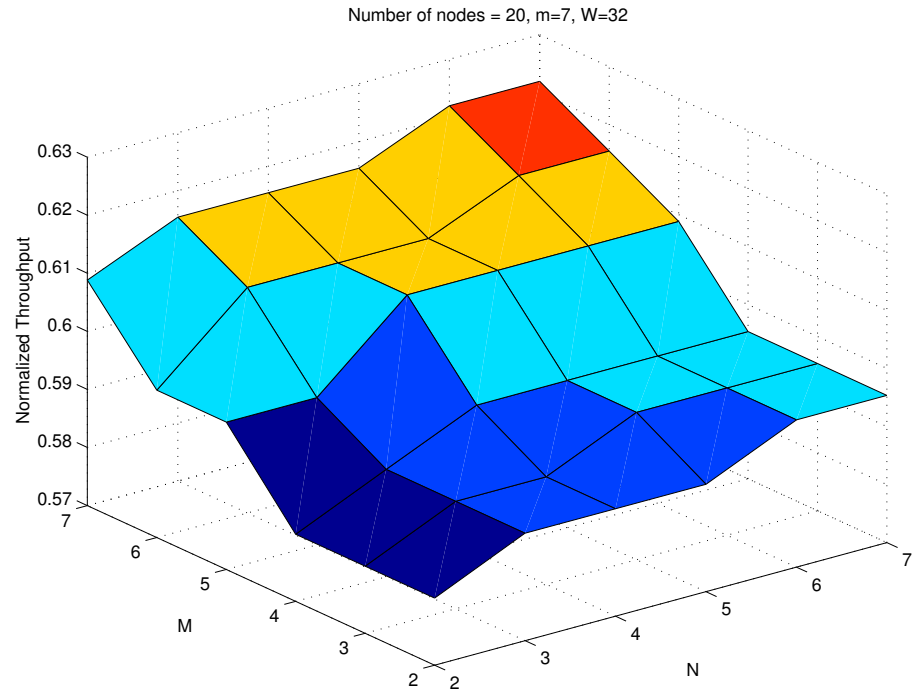

(b) 20 nodes

Figure 5: Normalized throughput versus threshold of consecutive transmission failures (N) and threshold of consecutive successful transmissions (M).

dynamic and their values depend on the network topology and on the density of nodes.

\section{Performance Evaluation}

In order to evaluate the performance of REFOT and compare it to other existing mechanisms such as classical REFOT (REFOT CL) [2], CARA [17] and DCF [11], we used metrics such as the fairness index $(F I)$, rate and throughput variations and mobility.

We use the fairness index $(F I)$ as a metric to evaluate the system fairness. The fairness index involves the relative throughput of nodes sharing a medium [9]. The fairness index of a system ranges from zero to one. Low values of the fairness index represent poor fairness among the competing nodes. Depending on the application and the number of senders, gaining higher fairness values is sometimes worthwhile even at the cost of reduced efficiency. In this paper with the introduction of the channel access probability, we define $F I$ as follows:

$$
F I=\frac{\left(\sum_{i=1}^{Z} Q_{d}^{J} r_{i}\right)^{2}}{Z \cdot \sum_{i=1}^{Z}\left(Q_{d}^{J} r_{i}\right)^{2}}
$$

where $Z$ denotes the total number of nodes in the given topology. The probability for each node to access a channel and the transmission rate are important parameters in (14) to calculate $F I$. Whilst $Q_{d}^{J}$ is defined for REFOT as in (3). For other schemes, such as for CARA and DCF, it can be calculated as follows :

$$
Q_{d}^{J}=\frac{1}{\left|\Phi_{J}\right|}
$$

where the number of neighbor nodes can be estimated by overhearing (i.e., we assume that a node can overhear the transmissions of other nodes, which fall into its transmission range properly). FI takes the value of one for the whole topology if each node, belonging to the network, has a probability to access the channel equal to one. Here, we want to clearly state that $F I$ varies with time. Since the rate changes in CARA and in REFOT, depending on the channel condition, a node may have different transmission rates at different times. Similarly, if the nodes, present in the network, are mobile, the probability to access the channel is dynamically estimated in our scheme, while this is not the case for CARA and the Classical RTS/CTS approaches. Therefore, FI may vary with time and other parameters, such as the channel quality, the number of competing nodes, etc.

To carry out this study, we used the network simulator NS2 [4] considering different scenarios. Moreover, several parameters related to these scenarios are taken into account such as the density of nodes, static and mobile nodes with different mobility models, different types of network traffic (e.g., UDP and TCP flows), different transmission ranges and the simulation time with different pause times.

The set of rates used for the rate adaptation is $R_{-} t h=$ $\{1 M b p s, 2 M b p s$,

5.5Mbps, $11 \mathrm{Mbps}\}$. The thresholds of consecutive transmission failures $(N)$ and consecutive successful transmissions $(M)$ are set to 3 and 4 , respectively. The simulations start with an initial rate $r=2 M b p s$. We considered a varying node speed, under the area of $800 \times 800 \mathrm{~m}^{2}$ with Two-Ray-Ground as propagation model. We varied the transmission range from $100 \mathrm{~m}$ to $250 \mathrm{~m}$ for different densities of nodes from 10 to 50 nodes. We use UDP flow with CBR (Constant Bite Rate) traffic and TCP flow randomly 
generated with NS tools (Cbrgen). The maximum packet size is 1460bytes. The routing protocol used is AODV (Ad hoc On-Demand Distance Vector) [22].

\subsection{Rate impact}

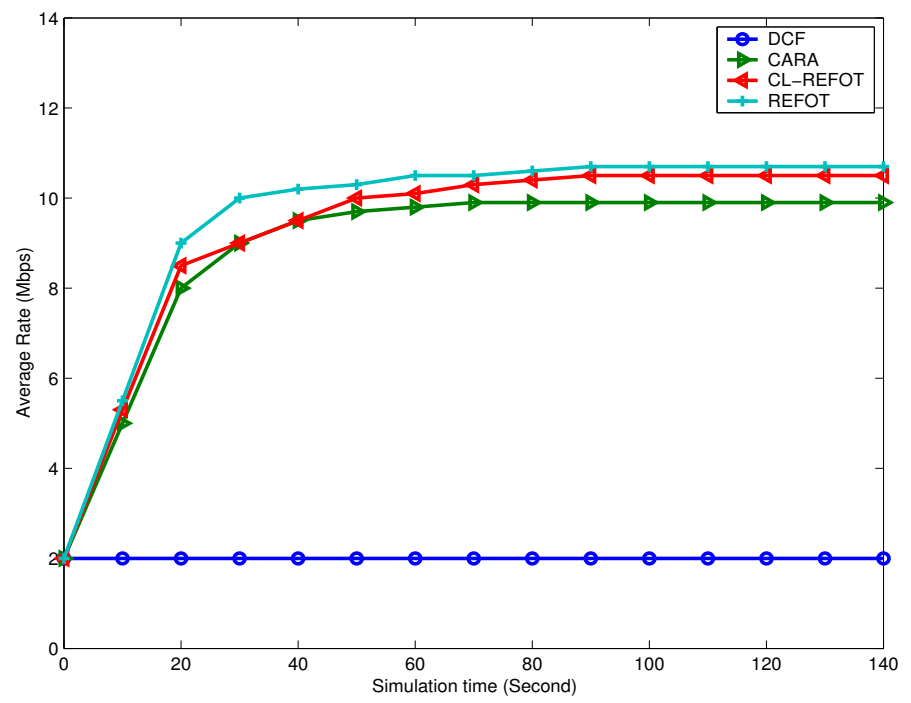

Figure 6: Average rate versus simulation time (UDP flow, with no mobility).

Fig. 6 illustrates the variation of the average rate during the entire simulation time, considering 20 static nodes with UDP flows. We notice that the rates obtained in case of REFOT are slightly better than $C A R A$ and the Classical REFOT due to the rate adaptation mechanism. The rate varies from $2 M b p s$ to $11 M$ bps. However, in case of $\mathrm{DCF}$, the rate remains constant, equal to $2 \mathrm{Mbps}$, due to the lack of rate adaptation mechanisms. In addition, these results show that the nodes have more consecutive successes than consecutive failures, so their rates can rapidly get stabilized. Moreover, we remark that the rate stability is reached 40 seconds after the start of the simulation. We can deduce that the possibility, offered by REFOT, to reduce the rate level of a node allows to adapt the rate level in the event of a critical situation, such as bad channel conditions.

In order to illustrate the mobility impact on the rate metric, we plot Fig. 7. This figure shows the average rate achieved during the simulation time in the case of 20 mobile nodes using UDP flows and moving at an average speed $2.5 \mathrm{~m} / \mathrm{s}$ following the RandomWayPoint mobility model. We notice that the average rate increases in case of REFOT compared to the Classical REFOT and CARA due to the mobility impact. Moreover, we remark that the rate is more stable when the nodes are static (with no mobility) as illustrated in Fig. 6. Knowing that the mobility may increase the probability of collision, the number of consecutive failures increases and then the rate decreases. That is why the rate is less stable than in the case of a static topology.

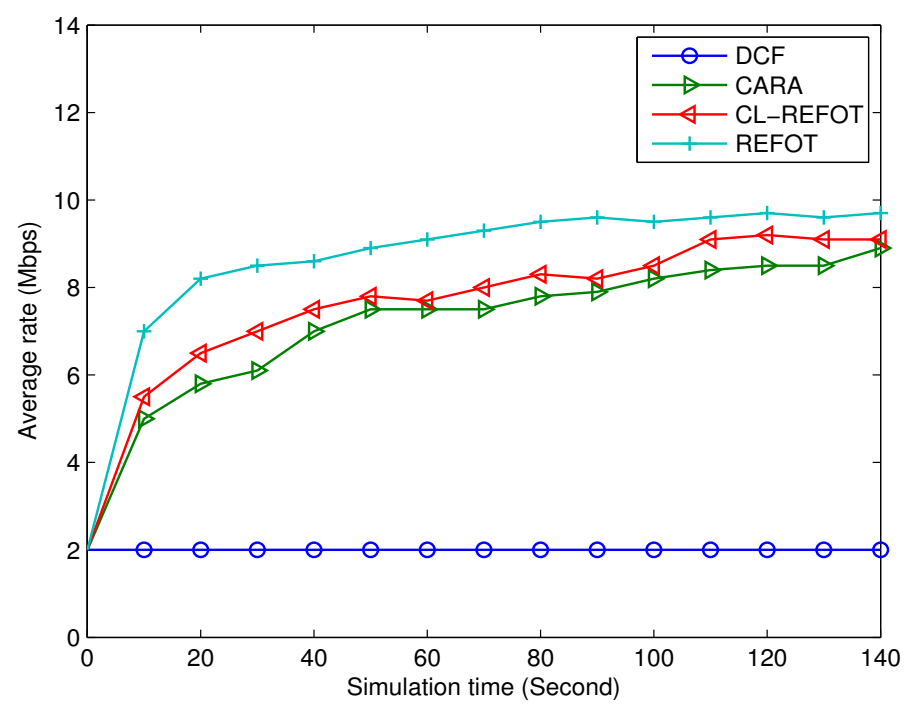

Figure 7: Average rate versus simulation time (Average mobility $2.5 \mathrm{~m} / \mathrm{s})$.

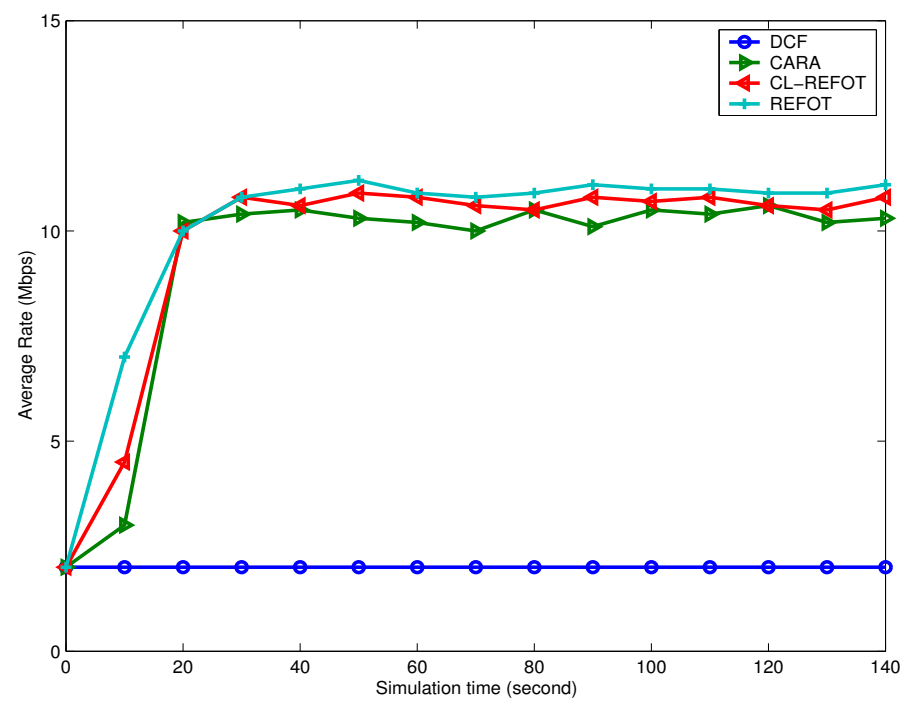

Figure 8: Average rate versus simulation time (TCP flow, static nodes). 
In order to study the connection impact, we consider TCP flows. The obtained results are plotted in Fig. 8. This figure shows the average rate according to the simulation time for 20 static nodes with a TCP flow generated randomly by Cbrgen tool. We observe that the rates obtained with 20 nodes in case of REFOT are not significantly different to those achieved by the Classical REFOT and CARA schemes. Intuitively, this performance is mainly due to the rate adaptation features of the three mechanisms. However, the rate reaches its maximum value quicker than with UDP flows.

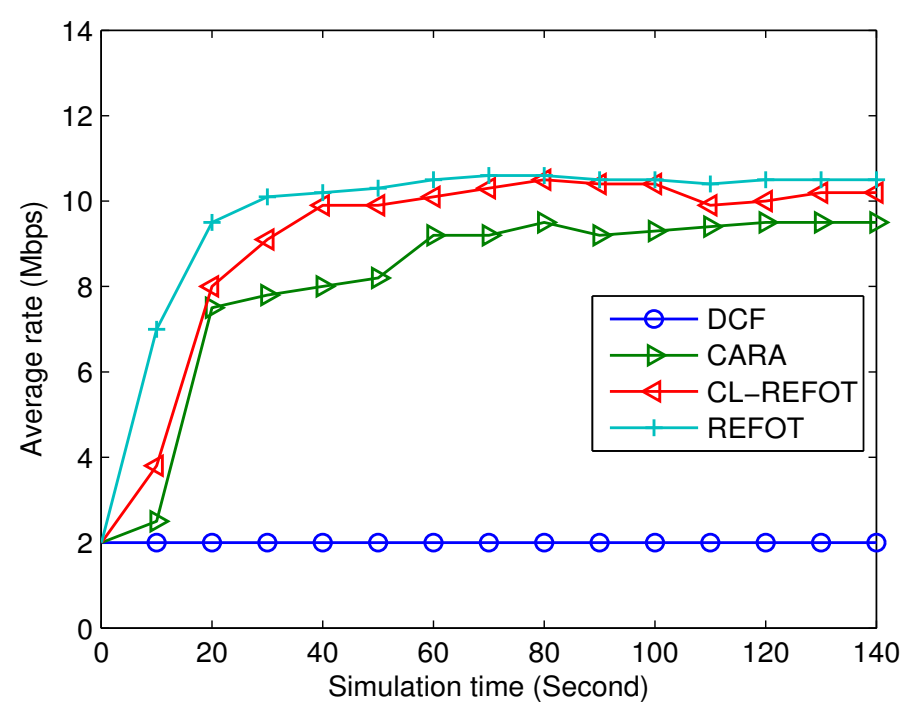

Figure 9: Average rate versus simulation time (TCP flow, Average mobility $2.5 \mathrm{~m} / \mathrm{s})$.

In order to illustrate the mobility impact on TCP flows, Fig. 9 plots the average rate variation during the simulation time for different mobile nodes moving at an average speed $2.5 \mathrm{~m} / \mathrm{s}$. We observe that the rates obtained in the case of REFOT are similar to the rates obtained with the Classical REFOT and CARA. However, the rate is almost the same as in the static case. The impact of mobility is offset by the stability of TCP connections. We can conclude that even with node mobility, the probability of collision and the number of consecutive failures when a connection-oriented protocol is used, are relatively low. We deduce that TCP mitigates the impact of mobility in terms of collided packets, so the rate is more stable.

\subsection{The fairness impact}

Ensuring fairness among competing nodes is one of REFOT goals. First, we study the impact of nodes density on the fairness index. We plot in Fig. 10 the fairness index $(F I)$ for different numbers of nodes in the network. We use UDP flows (randomly generated) and Random Way Point as mobility model with an average speed of $2.5 \mathrm{~m} / \mathrm{s}$. We notice that REFOT gives best performance in terms of fairness compared to other schemes particularly CARA and DCF. Furthermore, we remark that the fairness value

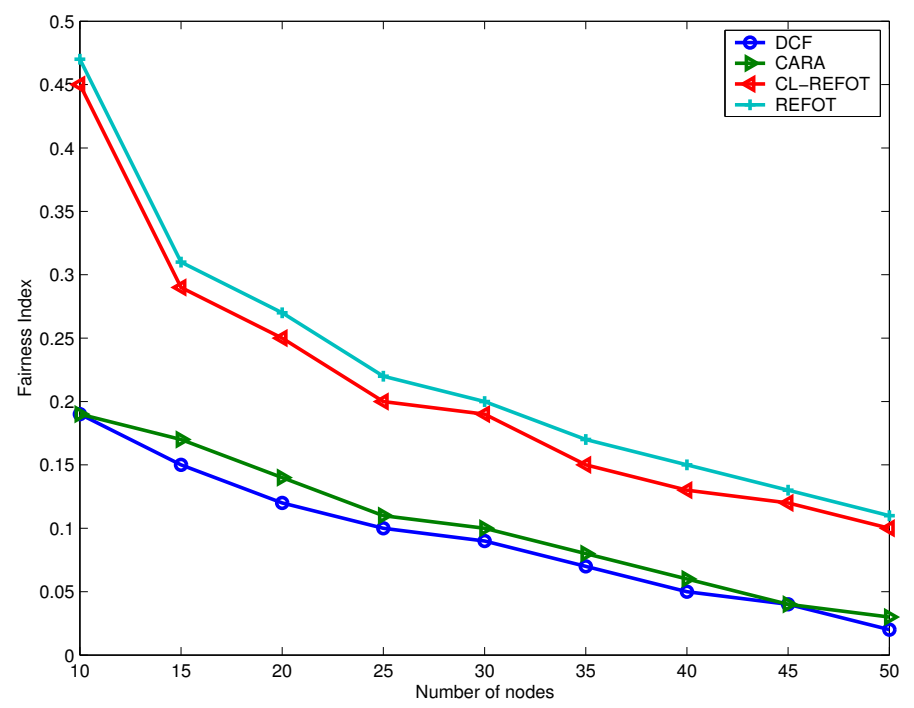

Figure 10: Fairness versus number of nodes (UDP flow, Average Mobility $2.5 \mathrm{~m} / \mathrm{s}$ ).

is approximately $40 \%$ greater than in $C A R A$ and DCF schemes. Since $C A R A$ and DCF do not take into account fairness for adapting the rate among contenders of a channel, both schemes exhibit lower values of the fairness index. We can deduce that when the number of nodes increases, the number of collisions may increase and the probability to access the channel will decrease. Then, the fairness index will decrease. Second, we study the transmission range impact on the fairness metric with the UDP and TCP flows and we plot the obtained results in Figs. 11(a) and 11(b). Fig. 11(a) shows that with 10 nodes having UDP flows, REFOT gives much better performance than CARA and DCF. The index is relatively stable and is approximately 0.4 greater than other schemes. However, in the case of TCP flows, we remark that the value of the Fairness Index $(F I)$ is higher when the transmission range is low. However, $F I$ decreases when the transmission range increases. It can be then deduced that the transmission range has an important impact on the fairness index in both cases of UDP and TCP flows, but the case of UDP flows gives better fairness than the case of TCP flows. We can conclude that, as the transmission range increases, the number of contenders in the channel increases, and hence the probability to access the channel decreases. Then the fairness index decreases when the transmission range increases.

Third, we focus on the mobility of nodes and its impact on the fairness parameter. Fig. 12 shows that with 10 nodes using randomly generated TCP flows, REFOT exhibits a better fairness compared to CARA and DCF. We notice that $F I$ of REFOT exceeds 0.5 which is not the case for CARA and DCF, where FI remains in the vicinity of 0.2 . Moreover, when the mobility of nodes increases, the fairness index is negatively affected. This performance is attributable to the probability to access the channel and the way to calculate it. We can conclude that REFOT out- 


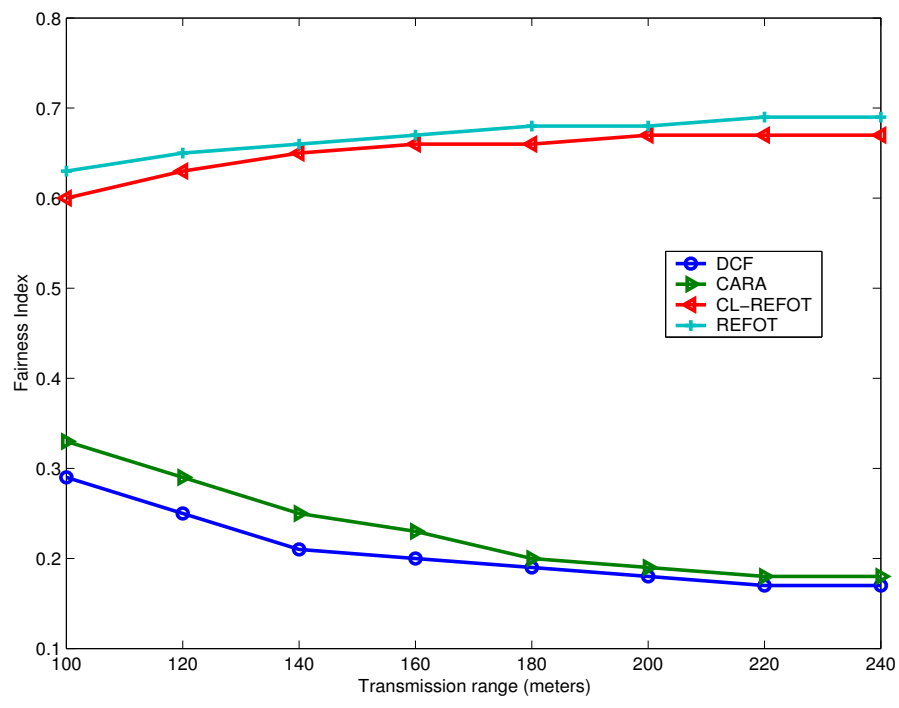

(a) UDP flow

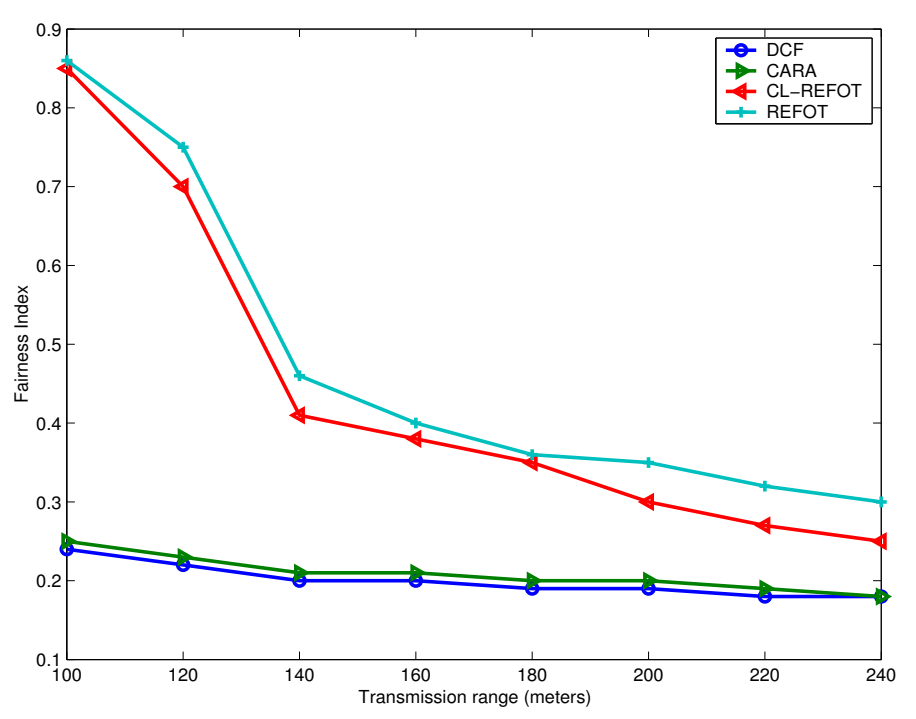

(b) TCP flow

Figure 11: Fairness index versus transmission range (10 nodes, Average mobility $2.5 \mathrm{~m} / \mathrm{s}$ ).

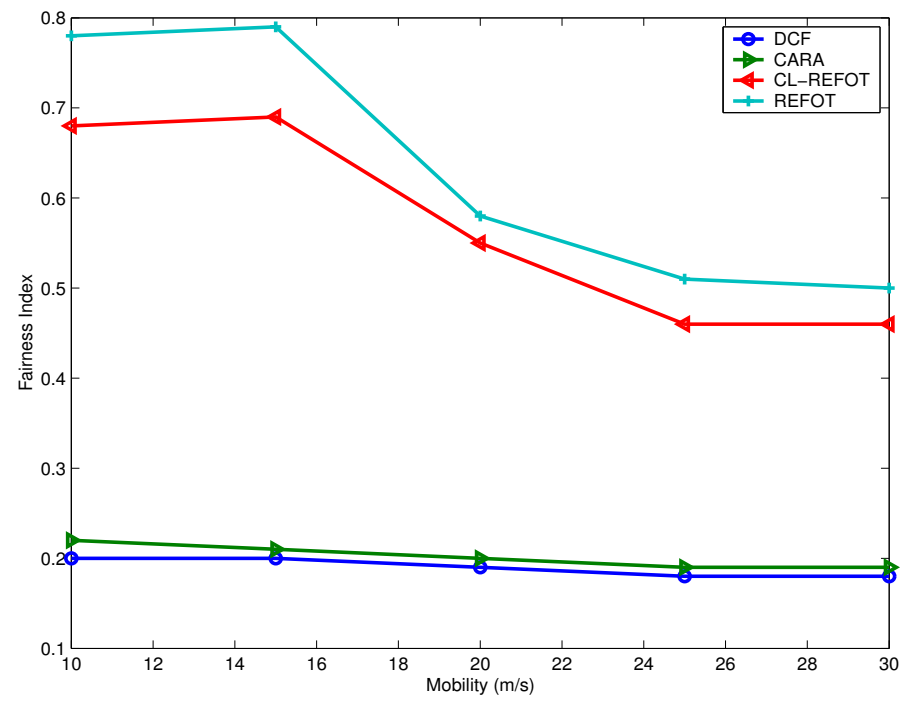

Figure 12: Fairness Index (FI) versus nodes mobility (10 nodes). performs the existing approaches, since CARA and DCF do not take into account fairness in rate adaptation.

\subsection{Impact on the throughput}

Fig. 13 plots average throughput achieved during different simulation times for 20 static nodes using UDP flows. We remark that our approach has nearly the same throughput as CARA and DCF.

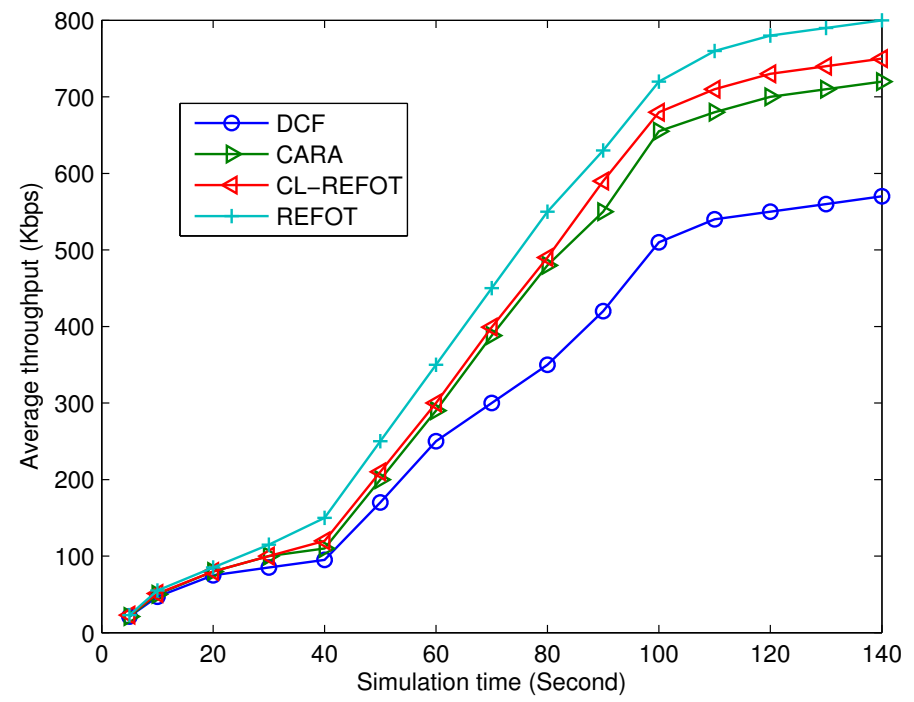

Figure 13: Throughput versus Simulation time (UDP flow).

Fig. 14 illustrates the throughput according to the simulation time for 20 mobile nodes using TCP flows. We notice that the throughput with REFOT stay close to the throughput with CARA mechanism. However, in case of DCF, the throughput is relatively low in comparison with 
CARA and our approaches (REFOT and REFOT CL). We remark that the throughput with TCP connections is higher than with UDP flows. Contrary to UDP (Fig. 13), we remark that due to the node mobility at the time instant 50s, the throughput of the simulated TCP flows decreases when the three schemes are used. However, the throughput starts increasing from the time instant 80s and gets stabilized from the time instant 100s. Therefore, we can deduce that taking into account the fairness in adapting the rate among contenders for a channel does not affect the throughput of the network.

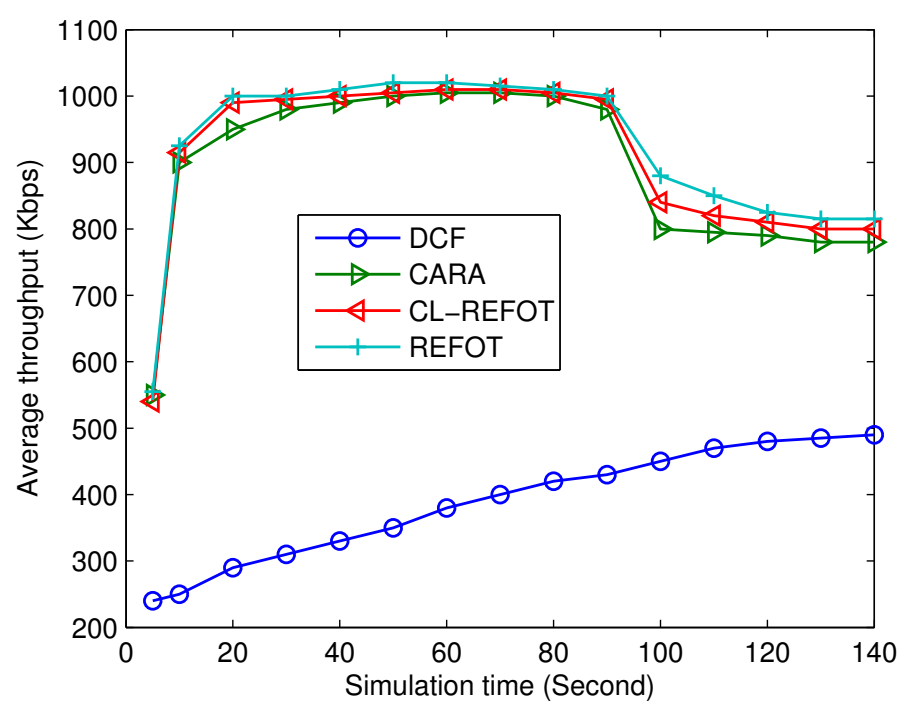

Figure 14: Throughput versus Simulation time (20 nodes, TCP flow).

\subsection{Mobility model impact}

The mobility model is an important parameter for the simulations. So, we will consider the Random Trip Mobility Model [21], because it is more realistic than the classical random way point of NS2 [4]. In order to illustrate the impact of different scenarios of mobility, we simulate three main mobility models [20]:

- Random Walk with Wrapping: This model is similar to the random waypoint, but at a trip transition instant, a node selects direction, trip duration, and numeric speed.

- Random Walk with Reflection: The difference between this model and the random walk with wrapping model is that whenever a node hits the boundary of the domain, it is not wrapped around, but reflected into the domain.

- Restricted Random Waypoint on a City Section: This is a particular instance of random waypoint on a general connected area. The domain is the union of line segments defined by the edges of a given space graph. The graphs used in the simulation represent real maps of several US cities.

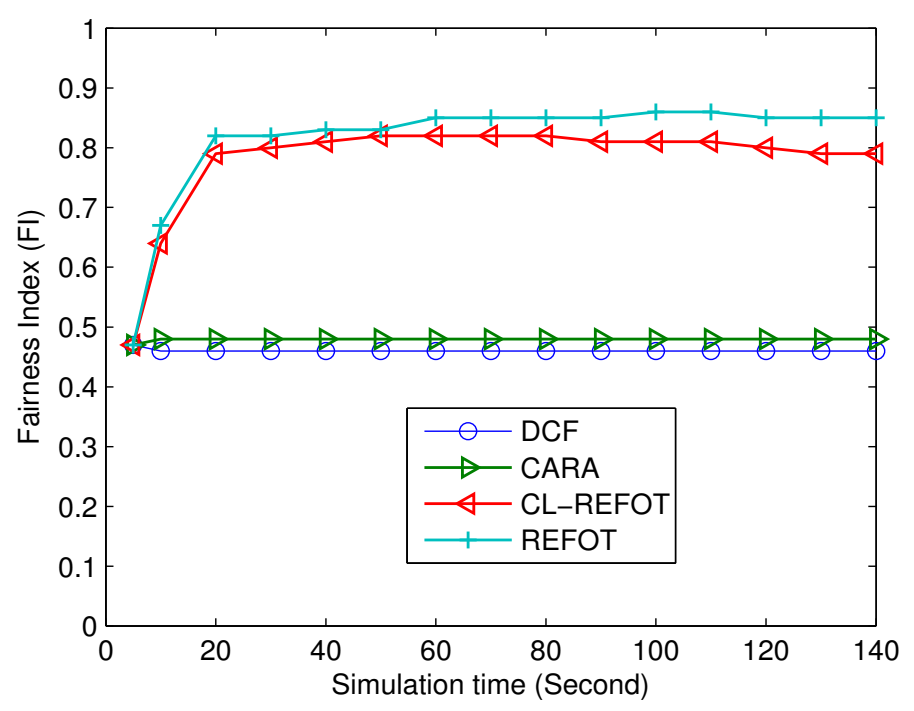

Figure 16: Fairness Index versus Simulation time (UDP flow, Restricted Random Waypoint on a City Section).

Fig. 15(a) plots the fairness index for different mobility speeds considering the Random Waypoint and Random Walk with Wrapping model. We remark that our schemes offer better performance than CARA and DCF in terms of fairness index. Moreover, we notice that with these two mobility schemes, the fairness index decreases less than the case of Random Waypoint (Fig. 12). Therefore, we can deduce that the negative impact of mobility is less important in this case. However, Fig. 15(b) illustrates the fairness index according to the mobility in the case of Random Waypoint and Random Walk with Reflection. The fairness index of REFOT is around $40 \%$ which is better than CARA and DCF. Furthermore, it is slightly better than in the previous case (Random Waypoint and random walk with wrapping).

Fig. 16 illustrates the fairness index according to the simulation time for 10 nodes with UDP flows. We used a Restricted Random Waypoint on a City Section. We used the map of West University place with an average speed of $5 \mathrm{~m} / \mathrm{s}[24]$. We notice that the fairness index is much better than with CARA and DCF. It is also much better than with the other schemes of mobility. The fairness index remains between 0.8 and 0.97 for REFOT and the Classical REFOT.

We notice that this mobility schemes give a better fairness index than the Random Waypoint. We will now study the impact on the throughput in order to show if taking into account the fairness in adapting the rate among all competing stations affects the throughput.

\section{Conclusion}

In this paper, we have proposed an analytical and mathematical model to adapt the transmission rate and to ensure relative fairness among ad-hoc competitors for com- 


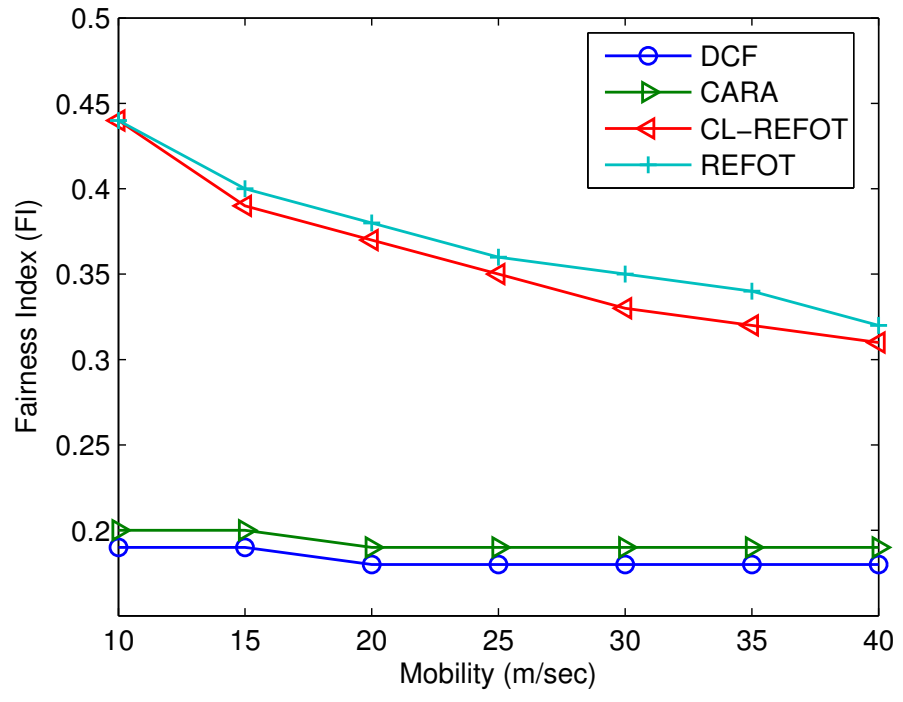

(a) case of Random Walk with Wrapping



(b) case of Random Walk with Reflection

Figure 15: Fairness versus mobility (10 nodes, UDP flow).

mon IEEE 802.11 channels without compromising the throughput in comparison with other existing approaches (e.g., CARA, DCF). The estimation of the probability to access a channel that is dynamically calculated, plays a key role in our approach. Fairness is defined while taking into account the probability to access a channel. We also introduced an analytical model using Markov chain to pattern our protocol. Through extensive simulations, we demonstrated that our proposed scheme outperforms existing ones in terms of different metrics and under different running conditions. Indeed, the proposed approach ensures fairness without compromising the throughput, even for highly mobile ad-hoc networks, such as vehicular ad hoc networks (VANETs). Hence our approach provides a balance between fairness in service and communication efficiency in terms of good throughput.

\section{References}

[1] Babu, A. V., Jacob, L., 2007. Fairness analysis of ieee 802.11 multirate wireless lans. IEEE Transactions On Vehicular Technology 56 (5), 3073-3088.

[2] Benslimane, A., Rachedi, A., Diwakar, D., 2008. Relative fairness and optimized throughput for mobile ad hoc networks. In: Proceedings of IEEE International Conference on Communications (ICC'2008), Beijing, China.

[3] Berger, S.-G., Duda, A., Gaudoin, O., Heusse, M., Rousseau, F., 2004. Fairness and its impact on delay in 802.11 networks. In: Proceedings of IEEE Global Telecommunications Conference (GLOBECOM'04), Dallas, Texas, USA.

[4] Berkeley, U., ISI, U., 1999. The network simulator ns-2. In: Part of the VINT project. Available from http://www/isi.edu/nsnam/ns.

[5] Berqia, A., Angoma, B., Mikou, N., Dehbi, Y., 2008. Fairness and qos in ad-hoc networks. In: Proceedings of IEEE Vehicular Technology Conference (VTC'2008), Marina Bay, Singapore.

[6] Bianchi, G., 2000. Performance analysis of the ieee 802.11 distributed coordianation function. IEEE Journal on Selected Areas in Communications 18 (3), 535-547.
[7] Bredel, M., Fidler, M., 2009. Understanding fairness and its impact on quality of service in ieee 802.11. In: Proceedings of International Conference on Computer Communications (INFOCOM'2009).

[8] Chevillat, P., Jelitto, J., Noll, B.-A., Truong, H.-L., 2003. A dynamic link adaptation algorithm for ieee 802.11a wireless lans. In: Proceedings of IEEE International Conference on Communications (ICC'2003), Anchorage, AK.

[9] Chiu, D., Jain, R., 1989. Analysis of the increase and decrease algorithms for congestion avoidance in computer networks. In: Computer Networks and ISDN Systems 17, 1-14.

[10] Holland, G., Vaidya, N., Bahl, P., 2001. A rate-adaptive mac protocol for multi-hop wireless networks. In: Proceedings of ACM Mo-biCom'01, Rome, Italy.

[11] IEEE802.11, 1999. Wireless lan medium access control (mac) and physical layer (phy) specifications. In: IEEE Std. 803.11.

[12] IEEE802.11e, 2005. Wireless lan medium access control(mac) and physical layer (phy) specifications. In: IEEE 802.11e Standard.

[13] IEEE802.11g, 2003. Wireless lan medium access control (mac) and physical layer (phy) specifications: Further higher data rate extension in the $2.4 \mathrm{ghz}$ band. In: Supplement to IEEE 802.11 Standard.

[14] Jain, R., Chui, D., Hawe, W., 1984. A quantitative measure of fairness and discrimination for resource allocation in shared systems. In: Digital Equipment Institution, Tech. Rep.

[15] Kamerman, A., Monteban, L., 1997. Wavelan-ii: a highperformance wireless lan for the unlicensed band. In Bell Labs Technical Journal 2 (3), 118-133.

[16] Khan, S., Mahmud, S. A., Loo, K.-K., Al-Raweshidy, H.-S., 2008. A cross layer rate adaptation solution for ieee 802.11 networks. Journal of Computer Communications 31 (8), 1638-1652.

[17] Kim, J., Kim, S., Choi, S., Qiao, D., 2006. Cara: Collisionaware rate adaptation for ieee 802.11 wlans. In: Proceedings of International Conference on Computer Communications (INFOCOM'2006).

[18] Kolara, V., Bharathb, K., Abu-Ghazalehc, N. B., Riihijarvid, J., 2011. The effect of contention in csma networks: Model and fairness protocol. Performance Evaluation 68 (9), 782-805.

[19] Kwak, B.-J., Song, N.-O., Miller, L. E., 2005. Performance analysis of exponential backoff. In journal of IEEE/ACM Trans. on Networking 13 (2), 343-355.

[20] Le Boudec, J.-Y., Vojnovic, M., 2005. Perfect simulation and stationarity of a class of mobility models. In: IEEE INFOCOM, 
Miami, FL, USA.

[21] PalChaudhuri, S., Le Boudec, J.-Y., Vojnovic, M., 2005. Perfect simulations for random trip mobility models. In: IEEE 38th Annual Simulation Symposium, Diego, CA, USA.

[22] Perkins, C. E., Belding-Royer, E. M., S., D., ???? Ad hoc on demand distance vector (aodv) routing. In: IETF RFC 3561.

[23] Sadeghi, B., Kanodia, V., Sabharwal, A., Knightly, E., 2002. Opportunistic media access for multi-rate ad hoc networks. In: Proceedings of ACM MOBICOM, Atlanta, Georgia, USA.

[24] Saha, A., Johnson, D., 2004. Modeling mobility for vehicular ad-hoc networks. In: Proceedings of the first ACM international workshop on Vehicular ad hoc networks. ACM New York, NY, USA, pp. 9192.

[25] Tan, G., Guttag, J., 2004. Time-based fairness improves performance in multi-rate wlans. In: Proceedings of the annual conference on USENIX ATEC '04.

[26] Tinnirello, I., Choi, S., 2005. Temporal fairness provisioning in multi-rate contention-based 802.11e wlans. In: In Proc. IEEE Int. Symp. Worlds Wireless Mobile Multimedia Netw.

[27] Wong, D. T.-C., Hoang, A., Tham, C.-K., 2010. Airtime fairness in a rate separation ieee $802.11 \mathrm{~b}$ mac. In: IEEE VTC Spring.

[28] Xi, Y., Kim, B.-S., Wei, J.-B., Huang, Q.-Y., 2006. Adaptive multirate auto rate fallback protocol for ieee 802.11 wlans. In: Proceedings of IEEE Military Communications Conference (MILCOM'2006), Washington, DC, US. 